March 2013, Volume 3, No. 1 Pages (41 - 59)

\title{
The Impact of Multiple Intelligences on Learning Outcomes in Fencing Curriculum
}

\section{Ghada Omar*}

\author{
* Lecturer, Faculty of Physical Education for Girls, Alexandria University
}

\begin{abstract}
This research aims to develop an educational program using multiple intelligences in fencing curriculum for students in Faculty of Physical Education for Girls and identify: impact of this program on cognitive achievement; skill performance, and emotional side; all for fourth grade students, Education Division in the fencing curriculum, Identify differences in learning outcomes for fencing curriculum according to intelligence patterns. Researcher used experimental approach. Research sample were (70) student divided randomly into (20) student of pilot study, (25) students of experimental group, (25) students of control group which taught in traditional way. Researcher used multiple intelligences indicator list, cognitive test, skill evaluation form and educational program based on multiple inelegance theory as research tools. Results revealed that: Teaching strategies based on multiple intelligences patterns and to experimental group have positive effect on knowledge acquisition and skills performance and the emotional side of fencing curriculum. Linguistic and kinesthetic intelligence patterns contributed in fencing curriculum cognitive achievement. Kinesthetic and visual spatial intelligence patterns contributed in fencing skill performance. Social intelligence pattern contributed in emotional side of fencing skills
\end{abstract}

\section{Introduction:}

$\mathrm{L}$ earning process occurs as a result of interaction between learning environment including curricula, teaching methods, and aids on one hand, and between the learner's preparations and his mental and personality traits on the other. That is why the teachers understanding and applying learning theories is one of the basic requirements for effective teaching, it helps teacher to choose suitable teaching methods and strategies which suits the learner, even so many teachers neglect this, as they focus on the scientific material only, without considering learners individual differences. (Osman Hassan, 2002:7)

Learners individual differences affect their temperament, interests, and preferred patterns of learning, which they learn faster through it, It is important that students consider their individual differences and their awareness, minds, need and reality (Kawsar Koujak , 2001: 353) (Osman Hassan:15).

Jaber Abdel-Hamid (1997) argue that teacher's positive work must be based on one or more learning theories, and that successful teaching lies in learning theory selection, (Jaber AbdelHamid,1997: 4). Researcher believes that choosing teaching method and theory appropriate to recipient make him positive participator.

This research adopt Howard Gardner's multiple intelligences theory, which has emerged in (1983) in a book entitled " Frames of Mind" by Howard Gardner, who started research on this subject in Harvard University, with group of researchers to explore human intellectual's potentials, Gardner viewpoint was that intelligence is a range of skills enabling the person to solve real problems in life, or add a new valued product in one or more of the cultural frames. (Gardner, 1993: 60)

Kawsar Koujak (1995), quoting from Gardner, that every individual is capable of knowing world around him through different ways and methods called human multiple intelligences, i.e. knowing the world through language, mathematical logic, spaces estimation, music thinking, using his body to solve problems, or in 
making some things, as well as in understanding the rights of others. and individuals divergence is a result for differences strength of each type of intelligence, and in method of these intelligences overlapping and interaction when solving a problem or doing something. (Kawsar Koujak, 1995: 205)

Gardner (1999) divides intelligence, according to this theory to seven basic patterns: linguistic, mathematical logical, spatial. kinesthetic, musical rhythm, social and personal intelligences, and these patterns show that intelligence is not monolithic; and difference between individuals not in their intelligence degree, but in the intelligence patterns, and patterns may not be equal in individual, but it is possible to strengthen weaknesses through training and teaching. (Gardner, 1999: 2)

Armstrong (2000) noted that some individuals have very high levels in some or most of these patterns, and others appear to have a severe shortage in most or some of these patterns, most individuals is located between these two points. (Armstrong,2000:1)

Multiple intelligences theory is one of most powerful influences behind education change throughout the modern world, offering us a scientific and flexible framework to achieve educational objectives. According to Abdel Meguid Nchoati (1996) multiple intelligences theory is useful and positive, especially in education field, As individuals differs in terms of their affiliations and beliefs, values and personalities, they also may differ in intelligence patterns which opens the way for educators to invest, develop and take care of all mental activities that these individuals owns. (Abdel Meguid Nchoati,1996: 115 116).

Multiple intelligences is a scientific and effective tool, where educational plans vary and help learners who have difficulty in understanding to learn, help teacher for deep understanding of each learner preference, skills, and abilities, and provide learners with variety learning ways suits their intelligence patterns, and thus make learners more interested in education. (Kieran Egam,2003: 4-5).

Multiple intelligences theory can be used to solve weaknesses of teaching methods, where it is characterized by modality in organizing and applying teaching innovations, and stimulate learner's inactive brain (Armstrong, 2000: 48).

Funderstanding foundation (2001) noted that multiple intelligences affects learning through three basic elements: Curriculum: as it make curriculum balance in terms of integration of the arts, social networking, and self-awareness; Teaching Methods: which should take into account intelligence patterns; Evaluation: where evaluation methods should take into account multiplicity of intelligences in addition to selfassessment methods that help individuals to understand their intelligence. (Funderstanding,2001: 2.)

Mohammad Yousuf (2002) defines learning style as "the way employed by student to acquire knowledge." It is noteworthy that each student has his own way of learning, and confirms the existence of more than one method of learning (Mohammad Yousuf, 2002:1).Saada Khalil (2005) argue that learners' styles are the basis for learning construction, and emphasizes the discovery of how people learn, how they recognize informations, how they use their knowledge to balance between learning styles and personal achievement. All this aiming to allow students to take responsibility for educating themselves with good pedagogical guidance (Saada Khalil,2005: 4).

Researcher is in the opinion that knowing student's intelligent pattern is of great importance to know his suitable education style; which may be used as basis for selection of fencing curriculum teaching strategies, which suits each intelligence type, where it is necessary to choose strategies that make student keen to learn and enjoy learning, allowing student to achieve the best results.

Modern fencing is a sport activity which has a sports, social, psychological, educational objectives, as it is practiced according to certain laws and regulations governing the confrontation between competitors without any intervention other than from fencing ethics, honor and honesty. (Fatnat Gabriel et al, 2012:21)

"Fredrek Semoi" argue that fencing is characterized by deception in confrontation, Fencer knowing his abilities and competitor appreciation are important during competition, 
It is motor intelligence sport as fencer use his intelligence in both offensive or defensive skills, which sould not be done randomly, but as a result of special mental capabilities enable him to achieve best levels. (Fredrek Semoi,1996: 15)

Fencing curriculum combines numerous experiences and activities offered by Faculty of Physical Education for Girls, under the supervision and guidance of lecturer aiming to improve some learning outcomes and to achieve educational goals.

Ahmed Hussein Allaqany, Aly Ahmad Algamal (1999) define learning outcomes as all learning aspects can be obtained by the learner, whether in one or several situations or as a result of curriculum study, variety of learning aspects is an evidence of teacher efficiency who was able to plan and implement these experiences (Ahmed Allaqany et al,1999: 202).

Nader Fahmy et al (1999) defines learning outcomes as the changes in learner behavior as a result of learning process, for example adding new information to learner, or acquire specific skills in specific field or develop certain concepts and trends. (Nader Fahmy et al,199: 131).

Zeinab Mohamed defines learning outcomes as learners final grasping as result of learning process, that determines the effectiveness of educational system towards fulfilling its objectives through the evaluation process, which help to identify weaknesses and strengths and overcome it (Zeinab Mohamed, 2000:56), While Abdul Azim Abdul Salam (2002) defines it as the output which teacher can through it determine assumptions and proposals that help to improve and develop learning process (Abdul Azim Abdul Salam,2002 :77).

Nowadays teaching need more strategies to help learners to enrich their knowledge and develop different mental skills, and multiple intelligences theory open the way for multiple teaching strategies which can be applied easily, according to this theory no specific teaching strategies set is able to work effectively with all learners at all times, due to multiplicity of learners' intelligence patterns and abilities, through these strategies it is possible to implement interaction and integration between students' intelligent patterns which help in achieving fencing learning objectives, hence it is necessary to provide many teaching strategies to give students the opportunity to use their intelligence patterns.

Even though multiple intelligences theory is important in identifying learning outcomes in fencing curriculum, studies addressed multiple intelligences in sports field are limited, within most important of these studies Nashaat Mohammed (2010), Ahmed Farouk, Mahmoud Hussein (2008) Mohamed Abdel-Aziz and Hoda Mohamed (2008) Ahmed Nabih (2007), Mohammed Ibrahim and Randy Abdul Aziz (2007), Wael Refai (2006), Ahmed Ali (2006), Manal Mohamed (2005), Van Sickle Jennifer Louise (2004), Hirschhorn, Douglas, and Kamin (2000) ), Mohammed Hamid (1991), and Awad Bstawe (1990). Results of these studies confirmed that multiple intelligences theory helps to learn and achieve better interaction between student and teacher and better students' active participation, which leads to acquisition and mastery of skills in the least possible time.

Based on the above, was the thinking in teaching fencing curriculum based on this theory referred to above. As most of previous studies and researches have focused on using multiple intelligences theory in classroom and applied practice, confirms that there is a strong relationship between multiple intelligences and learning process, use of the theory enabled learners to develop their individual capabilities, despite of the multiplicity and diversity of studies that focused on reality and application of multiple intelligences theory, researcher couldn't come across any study dealt with fencing teaching using this theory. The researcher stresses on necessity of multiple intelligences theory use and identify its impact on learning outcomes, this research is the first of its kind in fencing teaching based on foundations and principles of this theory that can contribute positively to fencing learning outcomes and even all the education areas in Faculty of Physical Education for Girls Alexandria, and she hopes that will encourage students to use their strengths as a springboard to take advantage of their intelligence pattern, which can have a large impact in the redevelopment and modernization of educational system in general. 


\section{Research objectives}

This research aims to develop an educational program using multiple intelligences in fencing curriculum for students in Faculty of Physical Education for Girls and identify:

1- The impact of the program using multiple intelligences on cognitive achievement of fourth grade students, Education Division in fencing curriculum.

2- The impact of the program using multiple intelligences on skill performance of fourth grade students, Education Division in fencing curriculum.

3- The impact of the program using multiple intelligences on emotional side of fourth grade students, Education Division in the fencing curriculum.

4- Differences in learning outcomes for fencing curriculum according to intelligence patterns.

\section{Hypotheses}

1- There are significant differences between experimental and control groups in post measurement of cognitive achievement in favor of experimental group.

2- There are significant differences between experimental and control groups in post measurement of skill performance in favor of experimental group.

3- There are significant differences between experimental and control groups in post measurement of emotional side in favor of experimental group.

4- There are significant differences

Table (1): Significance of differences between Experimental and Control group in Anthropometric measurements and motor abilities tests

\begin{tabular}{|c|c|c|c|c|c|c|}
\hline \multirow{2}{*}{ Measurement/test } & & \multicolumn{2}{|c|}{ Experimental $(\mathrm{n}=25)$} & \multicolumn{2}{|c|}{ Control $(n=25)$} & \multirow{2}{*}{$\mathrm{T}$ value } \\
\hline & & Mean & $\overline{S \mathrm{SD}}$ & Mean & $\overline{\mathrm{SD}}$ & \\
\hline \multirow{2}{*}{ Anthropometric } & Height & 153.773 & 6.005 & 154.882 & 5.158 & 1.347 \\
\hline & Wight & 67.038 & 8.467 & 68.415 & 9.368 & 0.967 \\
\hline
\end{tabular}

between the patterns of intelligence of students and learning outcomes in the fencing material.

\section{IV - Research plan}

Research Methodology: Researcher used experimental approach because of its relevance to research nature.

Research community : Research community represented in the fourth grade students, "Division of Education," Faculty of Physical Education Girls "Alexandria University" for the academic year (2011/2012) at econd semester, where total students number, according to college records is (85) students.

Research sample: After exclusion of absent students and players, students in the sample were (70) student has been divided randomly into (20) student of pilot study, (25) students of experimental group, (25) students of control group which taught in traditional way. In premeasurements homogeneous between experimental and control group was checked regarding : basic variables (chronological age height- weight) motor skills tests (appendix 2); Fencing curriculum cognitive test (appendix 3); Evaluation of fencing curriculum skills' performance level (appendix 4); multiple intelligences indicators list for fencing(appendix (5), Fencing curriculum trends scale (appendix 6)

1 - basic variables and motor skills: for both research group chronological age was recorded to nearest year, height to the nearest $\mathrm{cm}$, weight to the nearest $\mathrm{kg}$, most important physical abilities, and abilities tests obtained by referring to Mohammad Allawi, Nasreddin Radwan (1994), Mohamed Hassanein (1999), Ghada Omar (2009), Wafaa Darwish and Ghada Omar (2011) ), tests previously standardized in these studies. 


\begin{tabular}{l||l||l||l||l||l||l}
\hline & Age & 20.025 & 0.841 & 19.633 & 0.313 & 1.463 \\
\hline \hline \multirow{5}{*}{ Motor abilities tests } & Coordination & 5.845 & 1.375 & 5.643 & 1.385 & 0.497 \\
\cline { 2 - 6 } & Flexibility & 10.988 & 4.516 & 10.798 & 4.542 & 0.172 \\
\cline { 2 - 6 } & Agility & 11.433 & 0.667 & 11.214 & 0.833 & 1.124 \\
\cline { 2 - 6 } & Legs power & 1.644 & 0.227 & 1.64 & 0.318 & 0.875 \\
\cline { 2 - 6 } & Arms power & 6.207 & 0.812 & 5.918 & 0.713 & 0.772 \\
\cline { 2 - 6 } & Muscle endurance & 11.305 & 1.604 & 10.925 & 1.216 & 1.038 \\
\cline { 2 - 7 } & Accuracy & 2.47 & 0.432 & 2.375 & 0.392 & 0.881 \\
\cline { 2 - 7 } & Reaction speed & 1.551 & 0.352 & 1.532 & 0.342 & 1.677 \\
\hline \hline
\end{tabular}

Table (1) reveals no significant differences, so both groups are homogeneous.

2 - Cognitive test : Researcher built and designed the test in her doctoral dissertation (2009) (Ghada Omar, 2009) and re-standardized it by presenting to a group of experts (appendix 1) to ascertain its representing the goals which Table (2) Significance of differences between top and bottom quarters or cognitive test

\begin{tabular}{|c|c|c|c|c|c|}
\hline \multirow{2}{*}{ Test } & \multicolumn{2}{|c|}{ Top quarter $(n=5)$} & \multicolumn{2}{|c|}{ Bottom quarter $(n=5)$} & \multirow{2}{*}{$\mathrm{T}$ value } \\
\hline & Mean & SD & Mean & SD & \\
\hline Cognitive test & 85.526 & 3.851 & 63.158 & 3.871 & $10.379^{*}$ \\
\hline
\end{tabular}

* Significant at 0.05 level $(\mathrm{T}$ sig. $=2.18)$

Table (2) reveals significant differences between top and bottom quarters, which indicates that the test is valid and distinguishes between different levels.

Test reliability : Researcher applied and reapplied the test on sample of (20) students pther than main study sample in the period 12/2/2012 to $16 / 2 / 2012$ and calculated Cronbach alpha reliability coefficient to determine correlation between the first and second test.

Table (3) Reliability coefficient for cognitive test aspects

\begin{tabular}{|c|c|c|c|}
\hline Aspect & $\begin{array}{l}\text { Cronbach } \\
\text { alpha }\end{array}$ & Aspect & $\begin{array}{l}\text { Cronbach } \\
\text { alpha }\end{array}$ \\
\hline I (Physical aspect) & 0.962 & II (Technical-skill aspect) & 0.863 \\
\hline $\begin{array}{l}\text { III(Tactical-planning } \\
\text { aspect) }\end{array}$ & 0.896 & $\begin{array}{l}\text { IV (Teaching }- \text { learning methods } \\
\text { aspect) }\end{array}$ & 0.928 \\
\hline V (Law aspect) & 0.899 & & \\
\hline
\end{tabular}

Table (3) reveals that Cronbach alpha reliability coefficient are near to one which mean test high reliability.

3- Fencing skills performance evaluation form (appendix 4) : Researcher prepared this form to assess Fencing skills performance level by surveyed experts opinion (appendix 1) to make sure of students correct assessment, correct motor skills' phases performance, marks appropriate to each performance between $19 / 2 / 2012$ to $23 / 2 / 2012$, the necessary adjustments were made., the form represented to experts after adjustment, form applied on (20) 
students represent pilot study sample for checking validity and reliability.

Table (4) Significance of differences between top and bottom quarters of skill performance evaluation form

\begin{tabular}{|c|c|c|c|c|}
\hline \multicolumn{2}{|c|}{ Top quarter $(n=5)$} & \multicolumn{2}{|c|}{ Bottom quarter(n=5) } & \multirow{2}{*}{$\mathrm{T}$ value } \\
\hline Mean & SD & Mean & SD & \\
\hline 71.75 & 4.741 & 62.485 & 4.691 & $9.874^{*}$ \\
\hline
\end{tabular}

* Significant at 0.05 level $(\mathrm{T}$ sig. $=2.18)$

Table (4) reveals significant differences between top and bottom quarters, which indicate that the form is valid and distinguishes between different levels, Cronbach alpha reliability coefficient for the form were 0.913 which means high form reliability.

\section{4- Multiple Inelegancies indicators list:}

Objectives of list preparation: to document the relationship between fencing curriculum and indicators associated with each type of intelligence to be used in pre and post measuring within research procedures, as well as to determine the extent of activation of indicators employed in field of fencing by use of teaching strategies based on multiple intelligences theory.

\section{List preparation Steps:}

To determine list patterns and wording its indicators, researcher referred to what studied in first grade from knowledge, information, skills and objectives, get out a set of indicators for fencing curriculum which has been classified Table (5) and listed under each intelligence type, initial list included (69) behavioral indicator.

The researcher considered the following while wording indicators: Selecting clear and easy understanding verbs which easily can be associated to intelligence patterns; adopting principle of diversity in cognitive, practical and emotional aspects which indicators build upon it in fencing subject.

Standardizing the list

Content Validity: To verify list validity, researcher used content validity (experts validity), where she presented the list in its initial form to group of experts (appendix 1), then made all adjustments required by them , final list consisted of (64) indicator, distributed in six aspects related to intelligence types

Internal consistency: Researcher verified internal consistency by calculating the correlation of each indicator with its aspect (intelligence type)

Correlation coefficients for multiple intelligences indicators with their aspects

\begin{tabular}{|c|c|c|c|c|c|c|c|c|c|c|c|c|c|c|c|c|c|}
\hline $\begin{array}{l}\text { Indicator } \\
\text { No } \\
\text { Aspect }\end{array}$ & 1 & 2 & 3 & 4 & 5 & 6 & 7 & 8 & 9 & 10 & 11 & 12 & 13 & 14 & 15 & 16 & 17 \\
\hline $\begin{array}{l}\text { Linguisti } \\
\mathrm{c}\end{array}$ & $\begin{array}{l}0.51 \\
2 *\end{array}$ & $\begin{array}{l}0.61 \\
2 *\end{array}$ & $\begin{array}{l}0.45 \\
3 *\end{array}$ & $\begin{array}{l}0.54 \\
8 *\end{array}$ & $\begin{array}{l}0.48 \\
5^{*}\end{array}$ & $\begin{array}{l}0.51 \\
5 *\end{array}$ & $\begin{array}{l}0.65 \\
4 *\end{array}$ & $\begin{array}{l}0.57 \\
1 *\end{array}$ & $\begin{array}{l}0.49 \\
1 *\end{array}$ & $\begin{array}{l}0.52 \\
1 *\end{array}$ & $\begin{array}{l}0.75 \\
3 *\end{array}$ & $\begin{array}{l}0.60 \\
5^{*}\end{array}$ & $\begin{array}{l}0.5 \\
23\end{array}$ & $\begin{array}{l}0.61 \\
2 *\end{array}$ & $\begin{array}{l}0.52 \\
1 *\end{array}$ & $\begin{array}{l}0.87 \\
2 *\end{array}$ & $\begin{array}{l}0.65 \\
1 *\end{array}$ \\
\hline $\begin{array}{l}\text { Mathema } \\
\text { tical }\end{array}$ & $\begin{array}{l}0.48 \\
2 *\end{array}$ & $\begin{array}{l}0.48 \\
5^{*}\end{array}$ & $\begin{array}{l}0.52 \\
3 *\end{array}$ & $\begin{array}{l}0.66 \\
0^{*}\end{array}$ & $\begin{array}{l}0.54 \\
2 *\end{array}$ & $\begin{array}{l}0.60 \\
5 *\end{array}$ & $\begin{array}{l}0.66 \\
5^{*}\end{array}$ & $\begin{array}{l}0.75 \\
3 *\end{array}$ & $\begin{array}{l}0.48 \\
5^{*}\end{array}$ & $\begin{array}{l}0.51 \\
5^{*}\end{array}$ & & & & & & & \\
\hline $\begin{array}{l}\text { Visual } \\
\text { Spatial }\end{array}$ & $\begin{array}{l}0.78 \\
2 *\end{array}$ & $\begin{array}{l}0.51 \\
5^{*}\end{array}$ & $\begin{array}{l}0.78 \\
2^{*}\end{array}$ & $\begin{array}{l}0.57 \\
1 *\end{array}$ & $\begin{array}{l}0.62 \\
1 *\end{array}$ & $\begin{array}{l}0.65 \\
8 *\end{array}$ & $\begin{array}{l}0.51 \\
5^{*}\end{array}$ & $\begin{array}{l}0.65 \\
4 *\end{array}$ & $\begin{array}{l}0.61 \\
2 *\end{array}$ & & & & & & & & \\
\hline $\begin{array}{l}\text { Kinesthet } \\
\text { ic }\end{array}$ & $\begin{array}{l}0.52 \\
1 *\end{array}$ & $\begin{array}{l}0.61 \\
2 *\end{array}$ & $\begin{array}{l}0.52 \\
1 *\end{array}$ & $\begin{array}{l}0.48 \\
2^{*}\end{array}$ & $\begin{array}{l}0.48 \\
5^{*}\end{array}$ & $\begin{array}{l}0.72 \\
5^{*}\end{array}$ & $\begin{array}{l}0.62 \\
1 *\end{array}$ & $\begin{array}{l}0.46 \\
5^{*}\end{array}$ & & & & & & & & & \\
\hline
\end{tabular}




\begin{tabular}{|l|l|l|l|l|l|l|l|l|l|l|l|l|l|l|l|l|l|}
\hline Musical & 0.52 & 0.66 & 0.52 & 0.70 & 0.46 & 0.54 & 0.54 & 0.60 & 0.66 & 0.58 & & & & & & & \\
& $3^{*}$ & $0^{*}$ & $3^{*}$ & $8^{*}$ & $5^{*}$ & $8^{*}$ & $2^{*}$ & $5^{*}$ & $5^{*}$ & 6 & & & & & & & \\
\hline Social & 0.54 & 0.56 & 0.65 & 0.49 & 0.58 & 0.54 & 0.66 & 0.77 & 0.87 & 0.77 & & & & & & & \\
& $2^{*}$ & $4^{*}$ & $4^{*}$ & $6^{*}$ & 6 & 6 & $7 *$ & $5^{*}$ & $2^{*}$ & $9^{*}$ & & & & & & & \\
\hline
\end{tabular}

* Significant at 0.05 level $(\mathrm{r}$ sig. $=0.444)$

Table (5) reveals that all correlation coefficients between indicators and intelligence patterns are significant, indicating list validity.

Multiple Intelligences List's reliability:

Table (6) Reliability tests for multiple intelligences indicators list

\begin{tabular}{l|l|l|l|l|l|l}
\hline \hline Intelligence type & Linguistic & Mathematical & $\begin{array}{l}\text { Visual } \\
\text { Spatial }\end{array}$ & Kinesthetic & Musical & Social \\
\hline $\begin{array}{l}\text { Reliability } \\
\text { coeffecients }\end{array}$ & 0.856 & 0.879 & 0.961 & 0.961 & 0.975 & 0.856 \\
\hline \hline
\end{tabular}

Table (6) reveals that multiple intelligences indicators list have high reliability coefficients.

5 - Fencing curriculum trends' scale (appendix

6): Researcher has prepared student trends scale towards fencing curriculum, with 29 Statement to be answered on triple scale ( 3 agree, 2 to some extent, 1 not agree). Researcher presented Table (7) Trends scale validity and reliability coefficients

\begin{tabular}{l||l||l}
\hline \hline & Cronbach alpha & $\begin{array}{l}\text { Self validity (reliability's square } \\
\text { root) }\end{array}$ \\
\hline \hline Trends scale & 0.961 & 0.980 \\
\hline \hline
\end{tabular}

Table (7) reveals high validity and reliability for trends scale

Research groups' homogeneous in pre-measurement:

Table(8) Groups homogeneous in cognitive test

\begin{tabular}{l||l|l||l||l||l}
\hline \hline \multirow{2}{*}{ Aspect } & \multicolumn{2}{|l||}{ Experimental $(\mathrm{n}=25)$} & \multicolumn{2}{l||}{ Control $(\mathrm{n}=25)$} & \multirow{2}{*}{ T value } \\
\cline { 2 - 5 } & Mean & SD & Mean & SD & \\
\hline \hline I (Physical aspect) & 1.793 & 0.382 & 1.793 & 0.372 & 0 \\
\hline II (Technical-skill aspect) & 16.141 & 2.244 & 16.66 & 2.215 & 0.641 \\
\hline III(Tactical-planning aspect) & 8.947 & 1.323 & 9.869 & 1.362 & 1.645 \\
\hline IV (Teaching - learning methods aspect) & 18.493 & 3.038 & 18.914 & 1.578 & 0.398 \\
\hline V (Law aspect) & 8.722 & 1.401 & 7.938 & 0.931 & 1.639 \\
\hline \hline
\end{tabular}

T sig. at $0.05=2.011$

Table (8) reveals that there are no statistically significant differences between both groups in cognitive test. 
Table(9) Groups homogeneous in skill test results

\begin{tabular}{l||l|l||l|l||l}
\hline \hline \multirow{2}{*}{\multicolumn{1}{l||l||l||l||l|}{ Experimental $(\mathrm{n}=25)$}} & \multicolumn{2}{l||}{ Control $(\mathrm{n}=25)$} & \multirow{2}{*}{ T value } \\
\cline { 2 - 5 } & Mean & SD & Mean & SD & \\
\hline \hline Basic skills & 25.74 & 0.795 & 29.494 & 0.455 & 1.795 \\
\hline Simple attack & 20.588 & 0.872 & 19.44 & 0.599 & 1.689 \\
\hline Compound attack & 17.01 & 0.913 & 17.577 & 0.735 & 1.72 \\
\hline Skills total & 63.338 & 1.54 & 66.511 & 1.481 & 1.347 \\
\hline \hline
\end{tabular}

T sig. at $0.05=2.011$

Table (9) reveals that there are no statistically significant differences between both groups in skill test results.

Table(10) Groups homogeneous in trends scale

\begin{tabular}{l||l|l||l||l||l}
\hline \hline \multirow{2}{*}{ Variables } & \multicolumn{2}{|l||}{ Experimental $(\mathrm{n}=25)$} & \multicolumn{2}{l||}{ Control $(\mathrm{n}=25)$} & \multirow{2}{*}{ T value } \\
\cline { 2 - 5 } & Mean & SD & Mean & SD & \\
\hline \hline Trends scale & 57.572 & 0.918 & 52.345 & 0.476 & 1.478 \\
\hline \hline
\end{tabular}

T sig. at $0.05=2.011$

Table (10) reveals no statistically significant differences between both groups in trends scale.

Table(11) Groups homogeneous in multiple intelligences indicator list

\begin{tabular}{l||l|l||l||l||l}
\hline \hline \multirow{2}{*}{ Aspects } & \multicolumn{2}{l||}{ Experimental $(\mathrm{n}=25)$} & \multicolumn{2}{l||}{ Control (n=25) } & \multirow{2}{*}{ T value } \\
\cline { 2 - 5 } & Mean & SD & Mean & SD & \\
\hline \hline Linguistic intelligence & 32.5 & 4.78 & 31.56 & 4.67 & 0.711 \\
\hline Logical / Mathematical intelligence & 18.75 & 4.15 & 19.58 & 4.17 & 0.756 \\
\hline Visual / Spatial intelligence & 15.32 & 5.345 & 16.09 & 5.975 & 1.156 \\
\hline Bodily/ Kinesthetic intelligence & 21.98 & 3.471 & 22.19 & 3.581 & 1.007 \\
\hline Musical intelligence & 25.37 & 3.287 & 26.157 & 3.314 & 1.152 \\
\hline Social intelligence & 25.91 & 4.152 & 26.231 & 3.93 & 1.12 \\
\hline \hline
\end{tabular}

T sig. at $0.05=2.011$

Table (11) reveals no statistically significant differences between both groups in multiple intelligences indicators list..

\section{Research tools}

1 - Educational program using multiple intelligences (appendix 7, 8)

Program overall objective: Impact of multiple intelligences on fencing curriculum learning outcomes

\section{Bases of designing the program}

a - Program is based upon fencing curriculum overall objective.

b - Setting goals for cognitive achievement.

c - Setting goal for psychomotor side.

d - Program flexibility and its applicability in practice.

e- Program should achieve students' integrated preparation in terms of knowledge, skill and emotive sides. 
$\mathrm{f}$ - Considering students' individual differences,

$\mathrm{g}$ - Progression from easy to difficult.

$\mathrm{h}-$ Considering safety and security factors.

Educational Program designing steps using multiple intelligences : By referring to scientific literature and studies as Nashaat Mohammed (2010), Ahmed Farouk, Mahmoud Hussein (2008), Manal Mohamed (2005), Mohammed Hamid (1991), Awad Bstawe ( 1990) researcher was able to determine the following:

- How to prepare daily lessons in terms of daily lesson plan, planning lesson presentation, identify lesson objectives, identify teaching strategies, determine educational media, educational enrichment activities, and evaluation methods.

- The researcher took into account to include in fencing lesson plan lesson number, time, objectives and content, intelligence type, strategies used, teaching aids, procedures, enrichment activities in both theoretical and practical lessons.

- Theoretical lesson time is (90) minutes / one lecturer per week i.e. 12 lesson over 12 weeks.

- Practical lesson time: (5) minutes administrative work, (10) minutes general physical preparation (warm up), (10) minutes of special physical preparation for strengthen muscle to work with skill to be learned, (30) minutes educational part using multiple intelligences, (30) minutes practical part to master the skill to be learned using multiple intelligences, (5) minutes cool down to prepare body to return to natural state.

- Taking into account preparing students minds for scientific content at lesson beginning by recalling prior learning requirements and review it, giving students opportunity discover lesson subject, present lesson, raising their concern and motivation, present lesson objectives clearly, organize ideas and information.

- Lesson objectives are clear and not open to many meanings and interpretations, each objective relate to specific learning outcome.

- Considering Logical order in fencing curriculum content (appendix 7, 8)

- For each lesson strategies suit to fulfill its objectives determined, each lesson includes number of strategies correspond to students intelligence patterns, researcher diversified theses strategies as she viewed multiple intelligences patterns as different approaches to provide educational experience..

- Researcher used (brainstorming - encourage learners to discover things themselves imagination and perception - motor concepts musical memory activation - cooperative groups) strategies which can be applied within lesson allocated time, and could be flexible depending on classroom circumstances.

- Researcher considered selecting educational media which can be provided or produced within available facilities, and which also should suit lesson content.

- Researcher focused on determining educational enrichment activities to be precisely linked to educational objectives.

- To ensure fencing lesson effectiveness, researcher selected evaluation methods help to measure students progress in all curriculum objectives..

2 - Fencing curriculum cognitive test (appendix

$3)$ :The researcher checked the test on pilot sample of (20) student in order to determine suitable test time ; estimating test marks. Test time determined as average time taken by students and amounted to (45) minutes, researcher prepared model answer.

3 - Fencing Skills evaluation done through faculty staff committee using form prepared (appendix 4)

4- Emotional side evaluation done using trends scale prepared by the researcher (appendix 6)

Pilot Study aimed to test educational program usage (appendix 7,8), applied on sample of (20) students from research community other than main study sample, results were : appropriateness of educational program using multiple intelligences, availability of tools and suitable place for applying the research, validity and reliability of research tools.

Tuition place was unified for both groups in fencing hall.

Main study carried out in 2011/2012 second semester academic year on (08.03.2012) to 


\subsubsection{2)}

Pre- Measurements carried for both groups in basic variables, physical abilities, multiple intelligence list, Fencing cognitive test, skill evaluation form, Fencing trends scale in period from $12 / 02-03 / 04 / 2012$ )

Post measurements conducted after program application for both groups in cognitive test, skill evaluation form, trends scale at the period from $20 / 5 / 2012$ to $22 / 5 / 2012$

Statistical work: Researcher used arithmetic mean, standard deviation, Pearson correlation Table (12) Significance of differences between experimental and control group in cognitive test post measurement coefficient, Cronbach's alpha reliability coefficient, $\mathrm{T}$ test, multi linear regression.

\section{Results and Discussion:}

First hypothesis: There are significant differences between experimental and control groups in post measurement of cognitive achievement in favor of experimental group.

To validate the first hypothesis, researcher compared cognitive test average results between experimental group and control group in using $\mathrm{T}$ test.

\begin{tabular}{l||l|l||l||l||l}
\hline \hline \multirow{2}{*}{ Aspect } & \multicolumn{2}{|l||}{ Experimental $(\mathrm{n}=25)$} & \multicolumn{2}{l||}{ Control $(\mathrm{n}=25)$} & \multirow{2}{*}{ T value } \\
\cline { 2 - 5 } & Mean & SD & Mean & SD & \\
\hline \hline I (Physical aspect) & 2.15 & 0.371 & 1.83 & 0.211 & $2.53^{*}$ \\
\hline II (Technical-skill aspect) & 18.921 & 1.955 & 17.368 & 2.175 & $2.54^{*}$ \\
\hline III(Tactical-planning aspect) & 11.522 & 3.15 & 11.012 & 1.421 & $2.15^{*}$ \\
\hline IV (Teaching - learning methods aspect) & 21.315 & 4.132 & 19.832 & 1.521 & $4.21^{*}$ \\
\hline V (Law aspect) & 10.722 & 2.52 & 8.321 & 0.941 & $5.95^{*}$ \\
\hline \hline
\end{tabular}

* Significant at $0.05(\mathrm{~T}$ sign. $=2.011)$

Table (12) reveals existence of statistically significant differences between experimental and control groups in all cognitive test aspects in favor of experimental group.

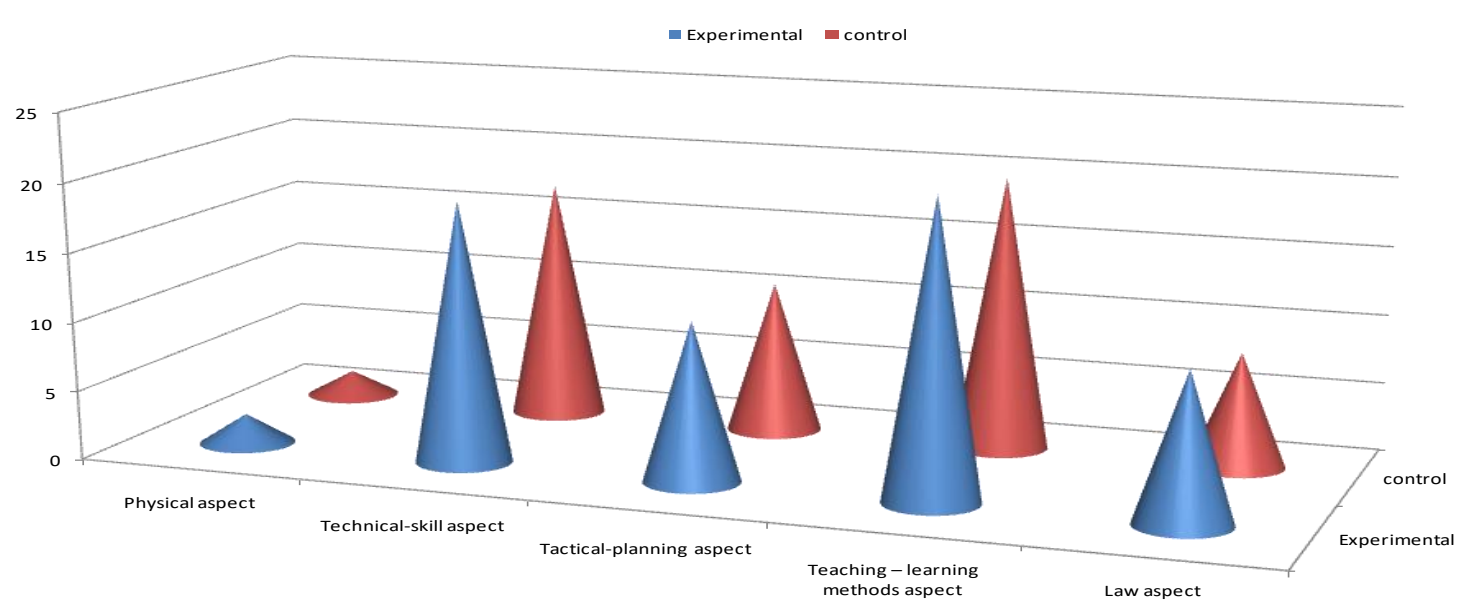

Fig (1) cognitive test post measurement for experimental and control groups

Table (13 Significance of differences between cognitive test pre and post measurement for experimental group

\begin{tabular}{l||l||l||l||l}
\hline \hline \multirow{2}{*}{ Aspect } & $\begin{array}{l}\text { Pre } \\
\text { measurement } \\
(\mathrm{n}=25)\end{array}$ & $\begin{array}{l}\text { Post } \\
\text { measurement } \\
(\mathrm{n}=25)\end{array}$ & $\begin{array}{l}\mathrm{T} \\
\text { value }\end{array}$ & $\begin{array}{l}\text { Improvement } \\
\text { rate (\%) }\end{array}$ \\
\hline
\end{tabular}




\begin{tabular}{|c|c|c|c|c|c|c|}
\hline & Mean & SD & Mean & SD & & \\
\hline I (Physical aspect) & 1.793 & 0.382 & 2.15 & 0.371 & $2.81 *$ & 19.911 \\
\hline II (Technical-skill aspect) & 16.141 & 2.244 & 18.921 & 1.955 & $3.92 *$ & 17.223 \\
\hline III(Tactical-planning aspect) & 8.947 & 1.323 & 11.522 & 3.15 & $4.16^{*}$ & 28.781 \\
\hline $\begin{array}{l}\text { IV (Teaching - learning methods } \\
\text { aspect) }\end{array}$ & 18.493 & 3.038 & 21.315 & 4.132 & $3.93 *$ & 15.26 \\
\hline V (Law aspect) & 8.722 & 1.401 & 10.722 & 2.52 & $4.98 *$ & 22.931 \\
\hline
\end{tabular}

* Significant at $0.05(\mathrm{~T}$ sign. $=2.064)$

Table (13) reveals existence of statistically significant differences between experimental groups pre and post measurements in all cognitive test aspects in favor of post measurement.

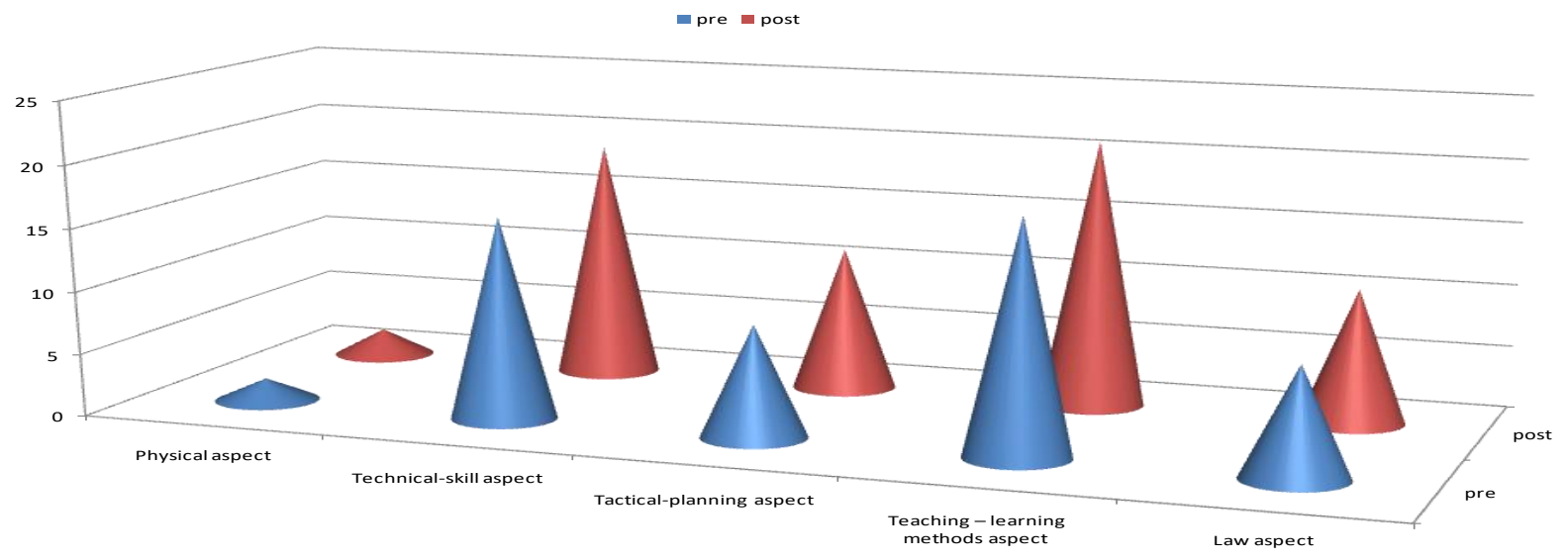

Fig (2) cognitive test pre and post measurement for experimental groups

Table (14) Significance of differences between cognitive test pre and post measurement for experimental group

\begin{tabular}{|c|c|c|c|c|c|c|}
\hline \multirow{2}{*}{ Aspect } & \multicolumn{2}{|c|}{$\begin{array}{l}\text { Pre measurement } \\
(\mathrm{n}=25)\end{array}$} & \multicolumn{2}{|c|}{$\begin{array}{ll}\text { Post } \\
(n=25)\end{array}$} & \multirow{2}{*}{$\begin{array}{l}\mathrm{T} \\
\text { value }\end{array}$} & \multirow{2}{*}{$\begin{array}{l}\text { Improvement } \\
\text { rate }(\%)\end{array}$} \\
\hline & Mean & $\overline{\mathrm{SD}}$ & Mean & $\overline{\mathrm{SD}}$ & & \\
\hline I (Physical aspect) & 1.793 & 0.372 & 1.83 & 0.211 & $2.691 *$ & 2.064 \\
\hline II (Technical-skill aspect) & 16.66 & 2.215 & 17.368 & 2.175 & $3.69^{*}$ & 4.25 \\
\hline III(Tactical-planning aspect) & 9.869 & 1.362 & 11.012 & 1.421 & 1.73 & 11.582 \\
\hline $\begin{array}{l}\text { IV (Teaching }- \text { learning } \\
\text { methods aspect) }\end{array}$ & 18.914 & 1.578 & 19.832 & 1.521 & 1.821 & 4.854 \\
\hline $\mathrm{V}$ (Law aspect) & 7.938 & 0.931 & 8.321 & 0.941 & $4.38 *$ & 4.825 \\
\hline
\end{tabular}

* Significant at $0.05(\mathrm{~T}$ sign. $=2.064)$

Table (14) reveals existence of statistically significant differences between control groups pre and post measurements in cognitive test physical, technical and law aspects only. 


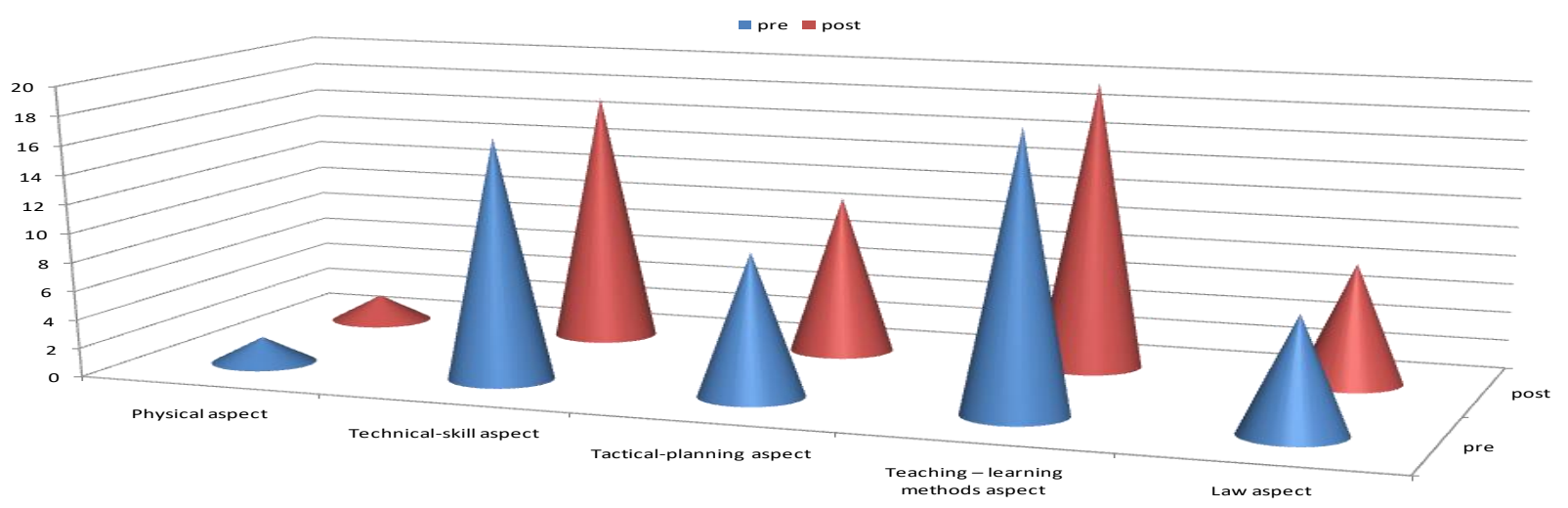

Fig (3) cognitive test pre and post measurement for control groups

\section{Discussion of first hypothesis results}

The results of tables (12), (13), (14) and figures (1), (2), (3) reveal existence of statistically significant differences between experimental and control groups in cognitive achievement in post measurement in favor of experimental group.

Researchers return superiority of experimental group to usage of teaching strategies based on multiple intelligences theory as it helps to teach each student with what suit her intelligence pattern. Unlike the traditional method, which followed with control group, where student is only listener.

Researcher also return the difference to encouraging experimental group students to do some activities in light of multiple intelligences which were helpful in study fencing curriculum content, each lesson has variety of methods and learning styles suiting different intelligence patterns, making students more interested in fencing. This what was recommended by Joyce Mary Ksicinsky (2000) that teachers must consider teaching strategies that are suits learner intelligence.

This result also consistent with Anderson (1998) study, which targeted impact of multiple intelligences activities strategies, methods of improving memory to retain information and knowledge, its results indicated by increase in students' marks, and also awareness of methods of remembering and awareness in different learning methods.

Researcher also return this superiority to different teaching strategies uses which represented in brainstorming, encourage learners to discover things themselves, motor concepts, imagination and perception, and this helped to provide curriculum knowledge and information in interesting and attractive manner, and helped to launch student mental abilities to its maximum.

In this regard, Kieran Egan (2003), and Ibrahim Abdullah (2005), Hassan Zaiton (2004) argue that these strategies accelerate learning process and characterized excitement, effective in thinking development, using these strategies is not related to particular time or place, help achieving effective learning.

Based on what mentioned above researcher can see that result of first hypothesis is consistent with results of Nashaat Mohammed (2010), Ahmed Farouk, Mohamed Hussein (2008), Manal Mohamed (2005) ), Mohammed Hamid (1991) Awad Bstawe (1990), which confirmed that application of multiple intelligences theory had a positive impact in classroom and give students positive trends about themselves and about their educational institutions, and had increased student incentive to achievement and success.

These results also proved that teaching using multiple intelligences strategies led to increase knowledge achievement, increase link between students and programs, behavior improvement , shorten times of tasks achievement, and motivate students to exert more effort and increase their pleasure when performing activities of multiple intelligences strategies, Thereby first hypothesis proved. 
Second hypothesis: There are significant differences between experimental and control groups in post measurement of skill performance in favor of experimental group.
Researcher verified second hypothesis by calculating $\mathrm{T}$ test value for differences between experimental and control groups in skill test results.

Table (15) Differences significance between experimental and control group in skill test post measurement

\begin{tabular}{|c|c|c|c|c|c|}
\hline \multirow{2}{*}{ Aspect } & \multicolumn{2}{|c|}{ Experimental $(n=25)$} & \multicolumn{2}{|c|}{ Control $(n=25)$} & \multirow{2}{*}{$\mathrm{T}$ value } \\
\hline & Mean & SD & Mean & SD & \\
\hline Basic skills & 2.15 & 0.371 & 1.83 & 0.211 & $2.53 *$ \\
\hline Simple attack & 18.921 & 1.955 & 17.368 & 2.175 & $2.54 *$ \\
\hline Compound attack & 11.522 & 3.15 & 11.012 & 1.421 & $2.15^{*}$ \\
\hline Skills total & 10.722 & 2.52 & 8.321 & 0.941 & $5.95 *$ \\
\hline
\end{tabular}

* Significant at $0.05(\mathrm{~T}$ sign. $=2.011)$

Table (15) reveals existence of statistically significant differences between both groups in skill test.

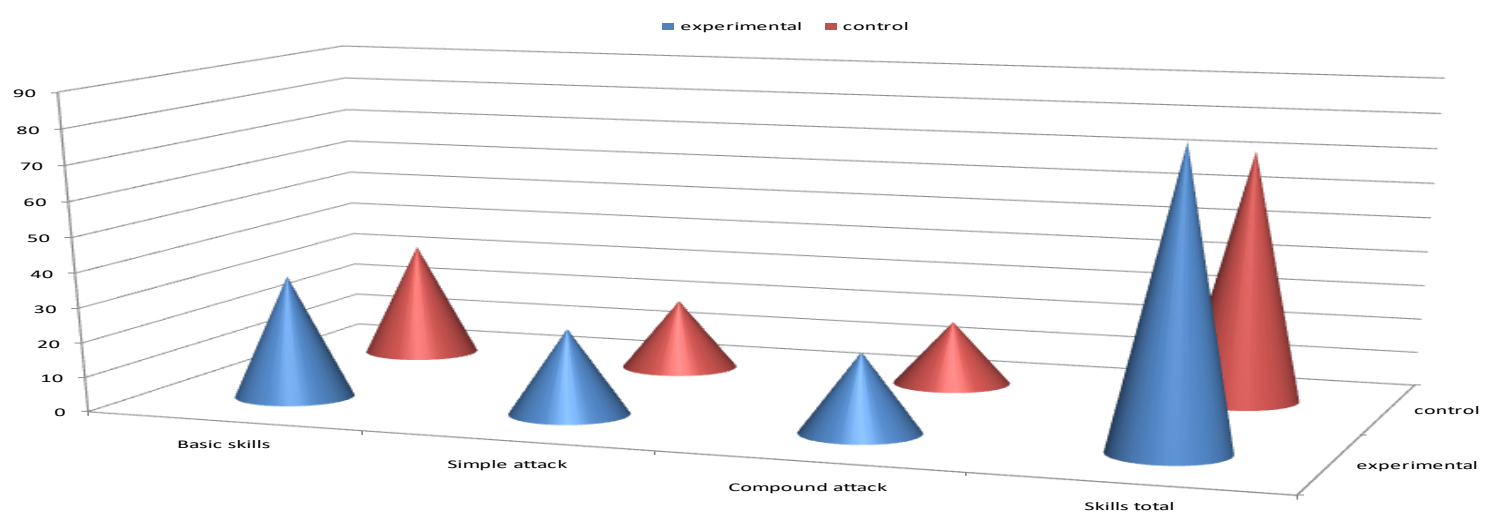

Fig (4) Skill test post measurement for experimental and control groups

\section{Discussion of second hypothesis results}

Table (15) and figure (4) results reveal existence of statistically significant differences between experimental and control groups in the skills performance level in favor of experimental group.

Researcher returns experimental group superiority to usage of varied teaching strategies based on multiple intelligences theory, which made planning of fencing practical lesson achieve balance in units presentation correspond to different intelligence patterns for each student, which made students more concerned to learn fencing.

Researcher also attribute this superiority to using multiple intelligence theory which made students more positive in classroom and more responsible in educational process and this was evidenced in keeping right performance of motor skills, they trained to correct errors as they arise, and this was also evidenced in increasing their marks.

Researcher also returns differences to varied teaching strategies used: brainstorming, encourage learners to discover things themselves, imagination and perception, motor concepts, musical memory activation, cooperative groups, which helped to take into account students' individual differences and their intelligence patterns, while in control group which followed the traditional method, depends on explanation and teacher performance students were only listeners and imitators.

Leslie Owen Wilson (1998) recommended using multiple intelligences theory as it considered an effective and universal tool allowing diversity of educational plans and lead learners with 
difficulty understanding to efficiently learn and independently.

Nader Ferjani (2001), Jolia Viens \& Silja kallenBach (2001) confirmed the importance of multiple intelligences theory for both teacher and learner and the possibility of applying it in all educational levels as it allow diversity of training and practices style cope with learners abilities, for learner theory is the way to discover his abilities and develop it in educational context, it also develops learner personal, social, vocational and cultural awareness and consciousness, Melanie Mitchell \& Michael Kernodle (2004) study results reveal that using multiple intilegence is effective in teaching complex tennis skills.

In light of the above researcher can see that Table (16) Differences significance between experimental and control group in trend scale post measurement these results agreed with, Ahmed Farouk, Mahmoud Hussein (2008), Manal Mohamed (2005), Awad Bstawe (1990) ,study results which confirmed that the application of multiple intelligences theory has an positive impact in preparation of students to improve their skills performance and achieve effective learning, Thereby second hypothesis proved.

Third hypothesis: There are significant differences between experimental and control groups in post measurement of cognitive achievement in favor of experimental group.

Researcher validated third hypothesis by calculating $\mathrm{T}$ test value for differences between experimental and control groups in skill test results.

\begin{tabular}{|c|c|c|c|c|c|}
\hline & \multicolumn{2}{|c|}{ Experimental $(n=25)$} & \multicolumn{2}{|c|}{ Control $(n=25)$} & \multirow{2}{*}{$\mathrm{T}$ value } \\
\hline & Mean & SD & Mean & SD & \\
\hline Trends scale & 67.113 & 5.125 & 54.133 & 6.752 & $4.244 *$ \\
\hline
\end{tabular}

* Significant at $0.05(\mathrm{~T}$ sign. $=2.011)$

Table (16) reveals existence of statistically significant differences between both groups in trends scale.

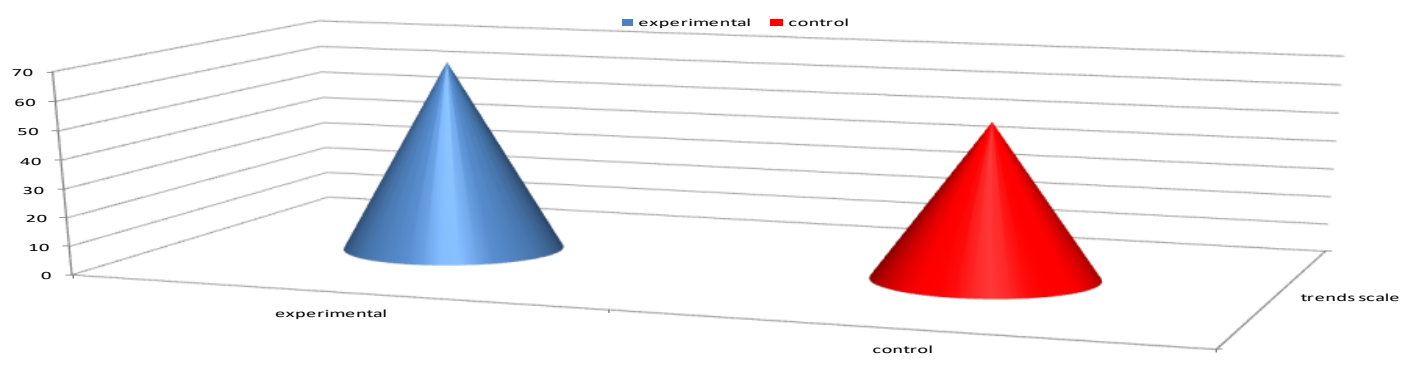

Fig (5) trends scale post measurement for experimental and control groups

\section{Discussion of third hypothesis results}

Table (15) and figure (5) results reveals existence of statistically significant differences between experimental and control groups in post measurement of emotional side in favor of experimental group.

Researcher return these differences to teaching strategies - used by experimental group- based on multiple intelligences theory that correspond to students individual differences and suit their different intelligence patterns, which led to social integration between students, And in turn raised group cohesion and give students the ability to perform with partner and at the same time note others performance.

Researcher also explain that by experimental group affection with teaching strategies to the degree that made each student is aware that her success reinforces and helps group success and achieving its objectives. This in turn helped to support cooperation spirit, encourage students during performance to discuss what they have learned and to exchange views and ideas among them, and helped to practice performance which is characterized by trust and leadership 
development

This is consistent with results of Nashaat Mohammed (2010), Mohammed Ibrahim and Randy Abdul Aziz (2007), Ahmed Nabih (2007), Wael Refai (2008) where all concluded that use of multiple intelligences theory had effective impact on improving the educational process, especially the emotional side.

This is confirmed by both Armstrong (2000), Kawsar Koujak(2001), Atef Abdel-Aziz (2003) where all argued that diversity in teaching strategies based on multiple intelligences theory has many benefits and positive educational implications in educational process i.e. selfconfidence, ability to understanding and communication, accept different points of view, leadership, unifying the efforts of students Table (17) Multiple regression for multiple intelligences contribution in cognitive test $\quad(n=25)$

\begin{tabular}{|l||l|l|l|l|l|l|l||}
\hline $\begin{array}{l}\text { Prediction indicators } \\
\text { Intelligence }\end{array}$ & $\mathrm{R}$ & $\mathrm{R}^{2}$ & $\begin{array}{l}\text { Contribution } \\
\%\end{array}$ & $\begin{array}{l}\text { Partial } \\
\text { regression }\end{array}$ & $\mathrm{T}$ & $\mathrm{F}$ & $\begin{array}{l}\text { Standard } \\
\text { Error }\end{array}$ \\
\hline \hline Linguistic intelligence & 0.484 & 0.235 & 23.5 & -0.544 & $2.698^{*}$ & $7.055^{*}$ & 9.491 \\
\cline { 2 - 8 } \\
$\begin{array}{l}\text { Bodily/ Kinesthetic } \\
\text { intelligence }\end{array}$ & 0.611 & 0.373 & 13.8 & 0.299 & $2.204^{*}$ & $6.549 *$ & 8.784 \\
\hline Constant & 111.277 & & & & \\
\hline
\end{tabular}

* Significant at 0.05 level (t sig. $=2.011, \mathrm{~F}$ sig. $=4.30)$

Previous table revealed that each of Linguistic intelligence, Bodily/ Kinesthetic intelligence contribute in cognitive test results with overall

percentage of $37.3 \%$, as $\mathrm{T}$ and $\mathrm{F}$ are statistically significant, so these two types are valid as predictor indicators for cogenitive test.

Table (18) Multiple regression for multiple intelligences contribution in skill test $\quad(n=25)$

\begin{tabular}{|l|l|l|l|l|l|l|l||}
\hline $\begin{array}{l}\text { Prediction indicators } \\
\text { Intelligence }\end{array}$ & $\mathrm{R}$ & $\mathrm{R}^{2}$ & $\begin{array}{l}\text { Contribution } \\
\%\end{array}$ & $\begin{array}{l}\text { Partial } \\
\text { regression }\end{array}$ & $\mathrm{T}$ & $\mathrm{F}$ & $\begin{array}{l}\text { Standard } \\
\text { Error }\end{array}$ \\
\hline $\begin{array}{l}\text { Bodily/ Kinesthetic } \\
\text { intelligence }\end{array}$ & 0.564 & 0.318 & 31.8 & -0.299 & $2.618^{*}$ & $10.705^{*}$ & 7.511 \\
\cline { 2 - 8 } & 0.659 & 0.435 & 11.7 & 0.157 & $2.136^{*}$ & $4.563^{*}$ & 6.989 \\
\hline Visual/spatial intelligence & 92.807 \\
\hline Constant & 97 & & & & \\
\hline
\end{tabular}

* Significant at 0.05 level (t sig. $=2.011, \mathrm{~F}$ sig. $=4.30)$

Previous table revealed that each of Bodily/ Kinesthetic intelligence , Visual/spatial intelligence contribute in cognitive test results with overall percentage of $43.5 \%$, as $\mathrm{T}$ and $\mathrm{F}$ are statistically significant, so these two types are valid as predictor indicators for skill test. 


\begin{tabular}{|l|l|l|l|l|l|l|l||}
\hline $\begin{array}{l}\text { Prediction indicators } \\
\text { Intelligence }\end{array}$ & $\mathrm{R}$ & $\mathrm{R}^{2}$ & $\begin{array}{l}\text { Contribution } \\
\%\end{array}$ & $\begin{array}{l}\text { Partial } \\
\text { regression }\end{array}$ & $\mathrm{T}$ & $\mathrm{F}$ & $\begin{array}{l}\text { Standard } \\
\text { Error }\end{array}$ \\
\hline \hline Social intelligence & 0.673 & 0.713 & 71.3 & -0.289 & $-2.932^{*}$ & $18.913^{*}$ & 12.123 \\
\hline Constant & 87.578 & & & & \\
\hline
\end{tabular}

* Significant at 0.05 level ( $\mathrm{t}$ sig. $=2.011, \mathrm{~F}$ sig. $=4.30)$

Previous table revealed that social intelligence, Visual/spatial intelligence contribute in cognitive test results with overall percentage of $71.3 \%$, as $\mathrm{T}$ and $\mathrm{F}$ are statistically significant, so this type valid as predictor indicators for trends scale.

\section{Discussion of fourth hypothesis results}

Table (17), (18), (19) results reveal existence of statistically significant differences between students intelligence patterns and learning outcomes in the fencing curriculum.

Researcher return linguistic intelligence high contribution in cognitive test results to usage of brainstorming strategy that correspond to linguistic intelligence pattern, which is one method of group discussion, which encourages members of the group to generate the largest possible number of innovative, creative ideas and proposals during relatively short period.

Masry Hanorh (1995) argue that what distinguishes this strategy is its application simplicity, do not require much effort or cost, requires shortest possible period, and its concrete results help to free imagination and push mind to its maximum energy. Fathi AbdelRahman (2002) adds that it is a large room for creativity and train students to respect other views; give more options and alternatives of solutions, accelerate learning process; in addition; it is characterized by excitement and thrill in developing effective thinking.

Researcher attribute contribution of kinesthetic intelligence in cognitive test results to usage of motor concepts strategy which affected by more than one intelligence type due to students individual differences.

Armstrong (2000) argues that this strategy focuses on the expression of certain information in a non-traditional manner, where learner translates specific concepts studied whether linguistic or logical to motor expressions.
Researcher in the opinion that linguistic, kinesthetic intelligences are the most important in positive impact towards fencing subject and acquiring them its skills..

Researcher returns contribution of Bodily/ Kinesthetic intelligence to using motor concepts strategy which allow using body parts in thinking, Gürel E, Tat M(2010) argue that people who are good in this intelligence type are able to learn and master sports skills easilly, she also returns visual spatial pattern in skill test results to usage of imagination and perception strategy where Abdul Latif Khalifa (2000) confirmed the importance of introducing imagination and its development through educational practices because of its importance in thinking development in general and to facilitate learning process and achieve educational goals, in particular.

Kieran Egan (2003) confirmed that educational material that will be taught through imagination facilitates the learner to remember information and not just store them and also contribute to a learning effect.

Researcher returns social intelligence contribution in emotional side (trends scale result) to usage of cooperative groups strategy which is effective in classroom.

Mohammed Ziad Hamdan (1998) indicates that this participation depend on common objectives for learning and educational tasks, as well as the roles they adopt during their interaction to reach the desired goals.

The cooperative groups strategy have many educational benefits and side effects in educational process, which summed up by Kawsar Koujak(1992), Atef Abdel-Aziz (2003) in the following: self-confidence, ability to understanding and communication, accept different points of view, leadership, elimination of learners isolation, dealing with others to accomplish tasks, reducing fear and anxiety. 
In researcher opinion cooperative groups strategy is appropriate to social intelligence pattern, because it can be adapted to suit all learners.

These results also in consistent with Dalia Zakria (2010) results which revealed that Bodily/ Kinesthetic intelligence, social intelligence and Visual/spatial intelligence are important for sports activities which require medium contact with competitor.

Hence it is clear educational process is influenced by different types of intelligence due to student individual differences, their needs, interests, and si educational process is an integrated process to achieve the balance and the desired objectives.

\section{Conclusions:}

1- Teaching strategies based on multiple intelligences patterns applied on experimental group have positive effect on knowledge acquisition and skills performance and the emotional side of fencing curriculum

2- Linguistic and kinesthetic intelligence patterns contributed in fencing curriculum cognitive achievement

3- Kinesthetic and visual spatial intelligence patterns contributed in fencing skill performance

4- Social intelligence pattern contributed in emotional side of fencing skills.

\section{Recommendations:}

- Conducting studies using teaching strategies based on multiple intelligences in other subjects.

- Studying the impact of use multiple intelligences theory on learning outcomes in other subjects.

- Using skill performance test in assessing faculty students.

\section{References}

1. Abdel-Azim Abdel-Salam Ferjani (2002): Education Technology and Development, Dar Gharib, Cairo. .(in Arabic)
2. Abdul Latif Khalifa, Shaker Abdul-Hamid (2000): Studies in curiosity, creativity and imagination, Al Gharib publishing and distribution, Cairo. .(in Arabic)

3. Abdul-Majid Nchoaty (1996): Educational Psychology. Dar Al-Furqan, Jordan. .(in Arabic)

4. Ahmed Ali Hagag Yusuf (2006): Sports administrator intelligent indicators as per multiple intelligences theory, unpublished Ph.D. thesis, Faculty of Physical Education of men, Helwan University. (in Arabic)

5. Ahmed Farouk Khalaf, Mahmoud Hussei, Mahmoud (2008): Effect of basics offensive tactical plans program on development tactical intelligence in basketball juniors, scientific journal of Physical Education and Sport, (56), Faculty of Physical Education for men, Helwan University. .(in Arabic)

6. Ahmed Hussein Allaqany, Aly Ahmad AlGamal (1999): Glossary of knowledge in educational curricula and teaching methods, Alm ElKotb, Cairo. (in Arabic)

7. Ahmed Nabih Ibrahim Mohamed (2007): Building emotional intelligence scale for athletes, scientific journal of Physical Education and Sports (49), Faculty of Physical Education of men, Helwan University. (in Arabic)

8. Anderson. Vb. (1998): Using Multiple Intelligence's to Improve Retention in Foreign Language Vocabulary study. ED 424745

9. Armstrong. T (2000): Multiple Intelligences in the Classroom. Alexandria. Virginia .2nd ed. ASCD

10. Atef Abdel-Aziz Abdel-Maksoud (2001): Effectiveness of using cooperative learning strategy on academic achievement and learning effect residual of students at agricultural high school. Journal of Research and technical education, (3), $18^{\text {th }}$ year, College of Education, University of Menoufia. .(in Arabic) 
11. Dalia Zakria Abbas zaid(2010): multiple intelligence model for individual and team sports' athletes, unpublished master thesis, faculty of physical education for girls, Alexandria university

12. Fathy Abdel-Rahman Jarwan (2002): Thinking education, concepts and applications, Dar ELfekr, Amman. .(in Arabic)

13. Fatnat Gabriel, Mahrosa Aly , Wafaa Darwish, Sabah Hanafi (2012): Fencing between theory and practice, 4th ed. Moltaka Elfeker Alexandria. .(in Arabic)

14. Fredric Semoi (1996): Fencing the world's oldest modern sportm the National broadcasting company, Colorado, USA,

15. Funderstanding

(2001):Multiple

Intelligences [online]

www.funderstanding.com/multiple-

intelligence.cfm

16. Gardner , H. (1993 ) Frames of Mind : The Theory of Multiple Intelligences. Tenth anniversary edition, New York : Basic books .

17. Gardner , H. (1999) : Intelligence Reframed : Multiple Intelligence for the 21_st Century . New York . Basic Books

18. Ghada Omar Mohamed Mohamud (2009): Effectiveness of proposed fencing curriculum in enhancing some learning outcomes for students in Faculty of Physical Education for Girls, unpublished Ph.D. thesis, Faculty of Physical Education for Girls, Alexandria University. .(in Arabic)

19. Hassan Hussein Zaiton (2004): teaching skills, vision in teaching implementation, $2^{\text {nd }}$ ed., Alam Elkotb, Cairo. .(in Arabic)

20. Hirschhorn, Douglas, Kamin(2000) : " The relationship between emotional intelligence and performance statistics of NCAA division 1 caliber baseball player " south Connecticut - state . University Dissertation abstracts international page 1691

21. Ibrahim Abdullah Al Humaidan, (2005): teaching and thinking, Center for Book Publishing, Cairo.(in Arabic)
22. Jaber Abdel-Hamid Jaber (1997): Intelligence and its measurements 10th ed., Dar AlNahda, Cairo. (in Arabic)

23. Jaber Abdel-Hamid Jaber (2003) Multiple Intelligences, understanding, develop and deepen, Dar ELfekr ElAraby, Cairo. .(in Arabic)

24. Joyce Mary ksicinski (2000) : Assessment of a Remedial Community College Cohort for Multiple Intelligences (D .A .I vol 61 02a p 0471)

25. Kausar Hussain Kojak (1992): Cooperative learning, teaching strategy achieved two goals, Journal of Educational Studies, Volume VII, Forty-third vol. .(in Arabic)

26. Kausar Hussain Kojak (2001): New trends in curricula and teaching methods, $2^{\text {nd }} e d$., Alm Elkotb, Cairo. .(in Arabic)

27. Kawsar Kojak, The Unschool Mind (1995): How Children Think: Howard Gardner \& How School Should Teach. Faculty of Education Journal, Helwan University, Cairo, January.

28. Kieran, Egam (2003): Memory Imagination and Learning, Connected by the Story Available: www.Ericdatabase1463245

29. Leslie Ower Wilson ( 1998 ):The Building Tool Room, Why are Teachers Drawn to Using Multiple Intelligence Theory in their Classrooms Available www.newhorizons.org/trm_lwilsonl. html

30. Manal Mohamed Zaki Elgendi (2005): Teaching developed rhythmic curriculum with strategies based on multiple intelligences theory and their impact on learning outcomes, unpublished Ph.D. thesis, Faculty of Physical Education for Girls, Alexandria University. .(in Arabic)

31. Masry Abdel-Hamid Hanorh (1995): Creativity and the road to the future, The future of Arab education, Volume I, Issue I. .(in Arabic)

32. Mohamed Abdelaziz, May Mohamed Awad (2008): Multiple Intelligences and its relationship to admission tests for students in Faculty of Physical Education for Girls, Education World magazine, Issue Twentyfourth, eighth year. .(in Arabic) 
33. Mohamed Sobhy Hassanein (1999): Measurement and Evaluation in Physical Education and Sports, $4^{\text {th }}$ ed., Dar Elfekr AlArabi ,Cairo. (in Arabic)

34. Mohammad Hassan Allawi, Nasreddin Radwan (1994): motor performance tests, 4d ed., Cairo, Dar Elfekr Al-Arabi. .(in Arabic)

35. Mohammad Yousuf (2002): learning styles, Teacher magazine cultural, educational, AlQattan Centre for Educational Research and Development , Gaza,/ Palestine. .(in Arabic)

36. Mohammed Awad Bstawe (1990): Intelligence relationship with some physical and motor skill variables in first class football players in Republic of Sudan, Unpublished Master Thesis, Faculty of Physical Education for men, Helwan University. .(in Arabic)

37. Mohammed Hamid Mohammed Shaddad (1991): Intelligence and its relationship to games outcome for Judo players, unpublished Master Thesis, Faculty of Physical Education for men, Helwan University. .(in Arabic)

38. Mohammed Ibrahim Mahmoud, Randy Abdul Aziz Hassan (2007): training program for tactical intelligence development for football juniors under 12 in 2007, scientific journal, Faculty of Physical Education for men, Alexandria University.(in Arabic)

39. Mohammed Ziad Hamdan (1998): Educational design, theory and practice, Dar Al Meera, Amman. .(in Arabic)

40. Nader Fahmy et al (1999): learning and classroom teaching, fourth edition, Dar Elfekr, Amman. .(in Arabic)

41. Nader Fergany (2001): Small brain myth , Mishkat Research Center, Egypt, Arab Council for Childhood and Development (Cairo), "Khtwa journal". .(in Arabic)

42. Nashaat Mohammed Ahmed Mansour (2010): emotional intelligence and its relationship with concern the results of sports competition and tournaments for emerging gymnastics, unpublished Ph.D. thesis, Faculty of Physical Education for Girls, Alexandria University. (in Arabic)
43. Osman Ali Hassan (2002): Teacher from functional to messianic, Journal of Education. Issue $143,31^{\text {st }}$ Year, Faculty of Law, University of Qatar. (in Arabic)

44. Saada Abdul Rahman Khalil (2004): Contemporary trends in learning and education: multiple intelligences, educational renewal. .(in Arabic)

45. Wael Ibrahim Radwan Rifai (2008): Building emotional intelligence scale for sports coach, scientific journal of Physical Education and Sport, Issue 48, Faculty of Physical Education of men, Helwan University. (in Arabic)

46. Wafa Mohammed Darwish, Ghada Omar (2011): Effect of mental visualization to improve performance skills of compound attack in fencing, scientific journal of Physical Education and Sport, Issue 43. .(in Arabic)

47. Zeinab Mohamed Amin (2000): technology Problems in education, Huda distribution, Minya. .(in Arabic) 